\title{
Performance on the Frontal Assessment Battery is sensitive to frontal lobe damage in stroke patients
}

Bruno Kopp ${ }^{1,2,3}$, Nina Rösser ${ }^{1,2}$, Sandra Tabeling ${ }^{1,4}$, Hans Jörg Stürenburg ${ }^{4}$, Bianca de Haan ${ }^{5}$, Hans-Otto Karnath ${ }^{5,6}$ and Karl Wessel $1^{1,2^{*}}$

\begin{abstract}
Background: The Frontal Assessment Battery $(F A B)$ is a brief battery of six neuropsychological tasks designed to assess frontal lobe function at bedside [Neurology 55:1621-1626, 2000]. The six FAB tasks explore cognitive and behavioral domains that are thought to be under the control of the frontal lobes, most notably conceptualization and abstract reasoning, lexical verbal fluency and mental flexibility, motor programming and executive control of action, self-regulation and resistance to interference, inhibitory control, and environmental autonomy.

Methods: We examined the sensitivity of performance on the $F A B$ to frontal lobe damage in right-hemisphere-damaged first-ever stroke patients based on voxel-based lesion-behavior mapping.

Results: Voxel-based lesion-behavior mapping of $F A B$ performance revealed that the integrity of the right anterior insula (BA13) is crucial for the $F A B$ global composite score, for the $F A B$ conceptualization score, as well as for the $F A B$ inhibitory control score. Furthermore, the $F A B$ conceptualization and mental flexibility scores were sensitive to damage of the right middle frontal gyrus (MFG; BA9). Finally, the FAB inhibitory control score was sensitive to damage of the right inferior frontal gyrus (IFG; BA44/45).

Conclusions: These findings indicate that several $F A B$ scores (including composite and item scores) provide valid measures of right hemispheric lateral frontal lobe dysfunction, specifically of focal lesions near the anterior insula, in the MFG and in the IFG.
\end{abstract}

Keywords: Frontal assessment battery (FAB), Abstraction, Fluency, Response inhibition, Anterior insula

\section{Background}

The Frontal Assessment Battery $(F A B)$ is a brief battery of six neuropsychological tasks that was specifically designed to assess frontal lobe function at bedside [1]. The historical roots of the six $F A B$ tasks can be found in the careful observation of dysexecutive behavior in patients with frontal lobe lesions, pioneered by Luria [2], Lhermitte, Pillon, and Serdaru [3], and others in the second half of the 20th century. The six $F A B$ tasks explore cognitive and behavioral domains of executive functioning that are thought to be critically dependent on the integrity of

\footnotetext{
* Correspondence: k.wessel@klinikum-braunschweig.de

${ }^{1}$ Cognitive Neurology, Technische Universität Braunschweig, Salzdahlumer

Str. 90, Braunschweig 38126, Germany

${ }^{2}$ Department of Neurology, Braunschweig Hospital, Salzdahlumer Str. 90, Braunschweig 38126, Germany

Full list of author information is available at the end of the article
}

the frontal lobes. The use of the $F A B$ is becoming increasingly popular for a variety of applications in neurology, most notably the early diagnosis of neurodegenerative dementing diseases such as the behavioral variant of fronto-temporal lobar degeneration (bvFTLD; $[4,5]$ ). The $F A B$ is easy to administer, requires less than ten minutes to complete, and is well accepted by patients. The reported psychometrics of $F A B$ reliability and validity are satisfactory [1], yet the anatomical correlation between $F A B$ scores and frontal lobe damage has never been established in studies of stroke patients. The claim that the $F A B$ yields indices of frontal lobe damage was derived from data obtained with similar tasks, but never from the $F A B$ tasks themselves [1].

In the present study, we investigated the sensitivity of performance on the $F A B$ to frontal lobe damage in

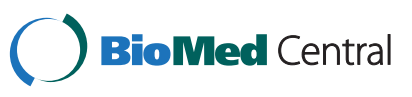


stroke patients using voxel-based lesion-behavior mapping (VLBM; [6-8]). In contrast to traditional overlap designs of neuropsychological patient groups [9], voxelbased lesion-behaviour analysis yields a sophisticated statistical approach to uncover brain-behaviour relationships. Voxelwise statistical analysis objectively estimates which brain regions indeed are associated with behavioral deficits without any prior categorization of stroke patients into, e.g., groups with more anterior versus more posterior brain damage. A major problem of the latter approach is that lesion boundaries are often overlapping in individual patients from different patient groups, thereby limiting the validity of such simple overlap group lesion studies (cf. [6]). Moreover, previous research on the behavioural effects of frontal brain damage often rested upon a comparison between groups of patients with lesions from many different etiologies (for a critical discussion see [10]).

To our knowledge, the VLBM method was applied for the first time to $F A B$ performance in stroke patients.

\section{Method}

\section{Subjects}

Thirty-one acute first-ever, right-hemisphere-damaged stroke patients with frontal lobe involvement participated in the study (see Table 1 for details). The logic behind the restriction to right-hemisphere-damaged stroke patients was to exclude patients with a paresis of the dominant right hand and/or with apraxia, possibly distorting task performance of these patients due to impaired

Table 1 Demographic and neuropsychological patient characteristics

\begin{tabular}{lccc}
\hline & $\boldsymbol{N}$ & $\boldsymbol{M}$ & $\boldsymbol{S D}$ \\
\hline Age & 31 & 59.61 & 10.31 \\
Sex & 31 & $19(\mathrm{~m}) / 12(\mathrm{f})$ & $/$ \\
Years of education & 31 & 12.24 & 2.26 \\
Handedness & 31 & 0.93 & 0.26 \\
CES-D [z] & 23 & 0.06 & 0.86 \\
MMSE [RS] & 31 & 27.42 & 2.26 \\
WST [z] & 31 & -0.36 & 0.83 \\
RWT - subtest s-words [PR] & 31 & 37.16 & 25.76 \\
RWT - subtest animals [PR] & 31 & 38.71 & 30.07 \\
MCST - N categories [RS] & 27 & 5.30 & 1.35 \\
MCST - N perseveration errors [RS] & 27 & 2.30 & 3.42 \\
\hline
\end{tabular}

Center for Epidemiologic Studies Depression Scale, CES-D [11]; German version); handedness: handedness ratio on the Edingburgh Handedness Questionnaire ([12]; -1 = strongly left-handed, $0=$ ambidextrous, 1 = strongly right-handed) . Mini-Mental-State-Examination (MMSE [13]); Modified Card Sorting Test (MCST [14]); Regensburger Wortflüssigkeits-Test [Regensburger Word Fluency Test] (RWT [15]); Wortschatz-Test [Vocabulary Test] (WST [16]).

Note: Sex: $m=$ male; $f=$ female; years of education: school and vocational education; $\mathrm{N}=$ number of subjects; $\mathrm{PR}=$ percentile rank; $\mathrm{M}=$ Mean; $\mathrm{RS}=$ Raw Score; SD = Standard deviation; $z=z$-score. motor functions. Further, left-hemisphere strokes might have hampered the capability to understand task instructions, due to the potential presence of sensory aphasia. ${ }^{a}$ Patients with diffuse or bilateral brain lesions due to traumatic brain injury, brain tumors, subcortical arteriosclerotic encephalopathy, or any other dementing disease were excluded. Patients had no history of psychiatric disease or alcohol or drug abuse. Further, patients with gross neurological defects (pronounced pain as reported by the patient, left homonymous hemianopia as revealed by clinical examination, hemispatial visual neglect) were also excluded to make sure that these symptoms did not interfere with task performance. ${ }^{\text {a }}$ Spatial neglect was diagnosed when a patient showed the characteristic clinical behaviour such as orienting toward the ipsilesional side when addressed from the front or the left and/or ignoring contralesionally located people or objects. Table 1 shows demographic and neuropsychological participant characteristics.

\section{Standard protocol approvals, registrations, and patient consents}

All patients gave their informed written consent to participate in the study, in accordance with the ethical standards of the Declaration of Helsinki (1964). Appropriate ethical approval for the study was obtained from the Ethics Committee at Technische Universität Braunschweig (Faculty for Life Sciences; Ref 37-2010).

\section{Test description, administration and scoring}

The $F A B$ consists of the following six tasks:

(1) Similarities (conceptualization). In this task, patients are required to identify the superordinate concept of two or more objects from the same semantic category. Specifically, patients were asked "In what way are the following objects alike?": (1) a banana and an orange, (2) a table and a chair, and (3) a tulip, a rose, and a daisy. Only category responses (fruits, furniture, flowers) were considered correct. If patients achieved three correct responses, the score was 3; if they achieved two correct responses, the score was 2; if they achieved one correct response, the score was 1 ; if they achieved no correct response, the score was 0 .

(2)Lexical verbal fluency (mental flexibility). This task requires the formation and exertion of self-organised cognitive strategies for efficient retrieval from semantic memory. It is well-documented in the neuropsychological literature that frontal lesions tend to decrease verbal fluency, particularly lexical verbal fluency $[17,18]$, and that in right-handed people, unilateral right frontal lesions are related to the presence of noticeable deficits in lexical verbal fluency 
[17]. Patients were instructed to say in 60 seconds as many words as possible beginning with the letter $S$, any words that came to their mind except surnames or proper nouns. If patients achieved more than nine words, the score was 3; if they achieved six to nine words, the score was 2; if they achieved three to five words, the score was 1 ; if they achieved less than three words, the score was 0 .

(3) Motor series (programming). This task requires the ability to program and execute a correctly ordered series of motor acts. Patients were asked to perform the Luria series 'fist, edge, palm' by initially copying the administrator three times, and then by repeating the series six times alone. If patients achieved six consecutive series by themselves, the score was 3 ; if they achieved at least three consecutive series on their own, the score was 2; if they failed at achieving at least three consecutive series alone, but achieved three when copying the examiner, the score was 1 ; otherwise the score was 0.

(4) Conflicting instructions (sensitivity to interference). This task challenges self-regulation in a behavioural interference paradigm by instructing patients to execute one action in response to the observation of a different action, thereby requiring the inhibition of imitative response tendencies $[3,19,20]$. Luria [2] had coined the term echopractic responses to signify his observation that patients with frontal lesions tend to display unintended imitative response tendencies. Patients were asked to hit the table once when the administrator hit it twice, or to hit the table twice when the administrator hit it only once. To ensure the patient had clearly understood the task, a practice trial was performed in which the examiner first hit the table once, three times in succession, and then twice, three more times. After the practice trial, the examiner completed the following series: $1-1-2-1-2-2-2-1-1-2$. If patients made no errors, the score was 3 ; if they made one or two errors, the score was 2; for more than two errors, the score was 1 , unless the patient copied the examiner at least four consecutive times, in which case the score was 0 .

(5)Go - Nogo (inhibitory control). Patients were told that now, when the examiner hit the table once, they should also hit it once, but when the examiner hit twice, they should do nothing. To ensure the patient had clearly understood the task, a practice trial was performed in which the examiner hit the table once, three times in succession, and then twice, three more times. After the practice trial the examiner completed the following series: 1-1-2-1$2-2-2-1-1-2$. If patients made no errors, the score was 3; for one or two errors the score was 2 ; for more than two errors the score was 1 , unless the patient copied the examiner at least four consecutive times, in which case the score was 0 .

(6) Prehension behavior (environmental autonomy). This task is designed to assess the tendency to activate patterns of behaviour that are involuntarily triggered by sensory stimulation, in some cases even against an explicit instruction not to show these activities. Following Dubois et al. [1], a particular sign of deficient environmental autonomy can be observed when the sensory perception (visual and/or tactile) of the experimenter's hand compels patients to take them (prehension behaviour). The patient's hands were placed palm up on the knees of the patient. The examiner touched both palms without saying anything. If the patient took the examiner's hands, the examiner tried again after having asked the patient, not to take his hands. If patients did not take the examiner's hands, the score was 3; if the patient hesitated and asked what to do, the score was 2; if the patient took the hands without hesitation, the score was 1 ; if the patient took the hands even after having been told not to do so, the score was 0 .

The $F A B$ global composite score was computed (range: $0 \ldots 18$ ) by summing up the six individual $F A B$ task scores.

\section{Lesion analysis}

Magnetic resonance imaging (MRI) was performed in 28 stroke patients and computed tomography (spiral CT) scanning was performed in three patients. The initial scanning was optionally repeated during the following days until the infarcted area became clearly demarcated. The mean time interval between lesion onset and the MRI scan that was used for the present analysis was 4.3 days $(S D=3.1)$; the mean time interval between time of lesion and CT scanning lasted 2.6 days $(S D=3.7)$. MRI scans were obtained on a $1.5 \mathrm{~T}$ echo planar imaging (EPI) capable system (Philips Intera, Philips Medical Systems, Best, The Netherlands). The MRI protocol used diffusion-weighted imaging (DWI, N=12) and T2weighted fluid-attenuated inversion-recovery imaging (FLAIR, $\mathrm{N}=16$ ). DWI was performed with a single-shot EPI spin echo sequence (25 axial slices; repetition times (TR), either 3690, 4000, 4452, 5060, 5300, or $6360 \mathrm{~ms}$; echo times (TE), either 90, 95, or $120 \mathrm{~ms}$; field of view (FOV), $230 \times 230 \mathrm{~mm}^{2}$; matrix $64 \times 64$ pixels; slice thickness, $5 \mathrm{~mm}$; gap, $5.5 \mathrm{~mm}$ ). The FLAIR sequences were acquired with 25 axial slices (thickness, $5 \mathrm{~mm}$ ) with an interslice gap of $5.5 \mathrm{~mm}$, a FOV of $220 \times 220 \mathrm{~mm}^{2}$, TR of either 4000, 5397, 5500, or $6000 \mathrm{~ms}$, and TE of either $89,91,100$, or $120 \mathrm{~ms}$. CTs were obtained on a spiral 
scanning system (Somatom Sensation 16, Siemens Healthcare, Erlangen, Germany) with a slice thickness of $3 \mathrm{~mm}$ infratentorial and $6 \mathrm{~mm}$ supratentorial and an in-plane resolution of $0.5 \times 0.5 \mathrm{~mm}$.

Lesion location was evaluated using MRIcroN software ([7], www.mricro.com). For patients with MRI scans, the boundaries of lesions were delineated directly on the individual MRI scans. Both the MRI scan and the lesion shape were then mapped into stereotaxic space using the normalization algorithm provided by SPM5 (www.fil. ion.ucl.ac.uk/spm/software/spm5/). Cost-function masking was employed [21] for determination of the transformation parameters.

In patients with spiral CT scans, lesions were drawn directly by an experienced neurologist (H.-O. K.; blinded for test performance) on the slices of a normalized $\mathrm{T}_{1}$ weighted template MRI scan from the Montreal Neurological Institute $(\mathrm{MNI})$ with a $1 \times 1 \mathrm{~mm}$ in-plane resolution, distributed with the MRIcroN toolset. Lesions were mapped onto the slices that correspond to MNI Z-coordinates $[-16,-8,0,8,16,24,32$, and $40 \mathrm{~mm}]$ by using the identical or the closest matching axial slices of each individual patient.

To evaluate the relationship between lesion location and performance on the $F A B$, a voxel-based lesionbehavior analysis was performed using the Liebermeister test implemented in the MRIcroN toolset [7]. The nonparametric Liebermeister test is performed on two binomial variables; it is a small-sample test for 2 by 2 tables. In the present context, one of the variables was 'lesion present' vs. 'lesion absent' in a particular voxel. Application of the Liebermeister test further requires patients to be assigned to two different groups based on a behavioural measure; given this, the Liebermeister test can identify voxels that when injured predict the presence of behavioral disturbance. The Liebermeister tests were based on median splits on the $F A B$ global composite score and on the six individual $F A B$ task scores (see Table 2 for the medians of the scores). Median splits were performed such that a " 0 " was assigned when task

Table 2 Neuropsychological results and Liebermeister test statistics (maximum Liebermeister z-score, critical Liebermeister z-score) over various FAB scores

\begin{tabular}{lllllll}
\hline FAB score & $\boldsymbol{M}$ & $\boldsymbol{S D}$ & $\boldsymbol{M d n}$ & $\boldsymbol{I Q R}$ & $\max . \mathbf{L z}$ & $\boldsymbol{z}_{\text {crit }}$ \\
\hline Global composite & 15.06 & 3.00 & 16.00 & 4 & $3.435^{*}$ & 3.113 \\
FAB 1 & 2.29 & 0.94 & 3.00 & 1 & $3.312^{*}$ & 2.966 \\
FAB 2 & 2.48 & 0.89 & 3.00 & 1 & $3.784^{*}$ & 3.341 \\
FAB 3 & 2.45 & 0.81 & 3.00 & 1 & 2.958 & 3.351 \\
FAB 4 & 2.74 & 0.45 & 3.00 & 1 & 3.302 & 3.466 \\
FAB 5 & 2.16 & 1.21 & 3.00 & 2 & $3.560^{*}$ & 3.143 \\
FAB 6 & 2.94 & 0.36 & 3.00 & 0 & 2.278 & 2.278 \\
\hline
\end{tabular}

Note: IQR $=$ inter-quartile range $\left(\mathrm{Q}_{75}-\mathrm{Q}_{25}\right) ;{ }^{*} p<.05$. scores fell below the median (i.e., "0-2" for the items scores), whereas a score of " 1 " resulted from task scores that equalled or outranged the median (i.e., "3" for the item scores). Test statistics are maximum Liebermeister $\mathrm{z}$-score $(\mathrm{Lz})$ and critical Liebermeister $\mathrm{z}$-score $\left(\mathrm{z}_{\text {crit }}\right)$; Lz $>\mathrm{z}_{\text {crit }}$ indicates that there were voxels that when injured predicted the presence of behavioral disturbance.

Only voxels that were damaged in at least three patients were included in the analysis ( $N=150.132$ voxels). We controlled for multiple comparisons using permutationbased thresholding using 4000 iterations, as advocated in $[7,22]$. All results presented survived a $5 \%$ permutationbased false positive probability threshold.

\section{Results}

\section{Neuropsychological test results on the FAB}

Table 2 summarizes the performance of the patients on the $F A B$. The average $F A B$ global composite score amounted to $M=15.06(S D=3.0)$. Task difficulty differed between the six $F A B$ tasks, with $F A B$ environmental autonomy being the easiest task and $F A B$ inhibitory control being the most difficult task. The $F A B$ conceptualizing score, the $F A B$ mental flexibility score, and the $F A B$ inhibitory control score showed relatively large variability compared to the $F A B$ motor programming score, the $F A B$ interference score, and the $F A B$ environmental autonomy score.

Table 2 also summarizes the results obtained with the nonparametric Liebermeister test over all $F A B$ scores ( $F A B$ global composite score and the six individual $F A B$ task scores) to identify whether or not there were voxels that, when injured, were associated with the presence of behavioral disturbances on the $F A B$. Statistical significance was found for the $F A B$ global composite score, the $F A B$ conceptualizing score, the $F A B$ mental flexibility score, and the $F A B$ inhibitory control.

\section{Lesion analyses: lesion overlap}

Figure 1 shows overlay lesion plots of all thirty-one patients in eight axial slices of a standard brain (i.e., in MNI space). Inspection of Figure 1 reveals that the maximum lesion overlap occurred in the right prefrontal cortex (PFC) where up to twelve patients showed overlapping lesions in single voxels.

\section{Lesion analyses: FAB global composite score}

Figure 2 displays the results of a lesion subtraction analysis for global composite score. Figure 2A shows the overlay lesion plot of those patients who achieved a $F A B$ global composite score below the median $(M d n=16)$. The overlay lesion plot of those patients who achieved a $F A B$ global composite score equal to or above the median is presented in Figure 2B. Figure 2C displays the results of a lesion subtraction analysis (patients below the 


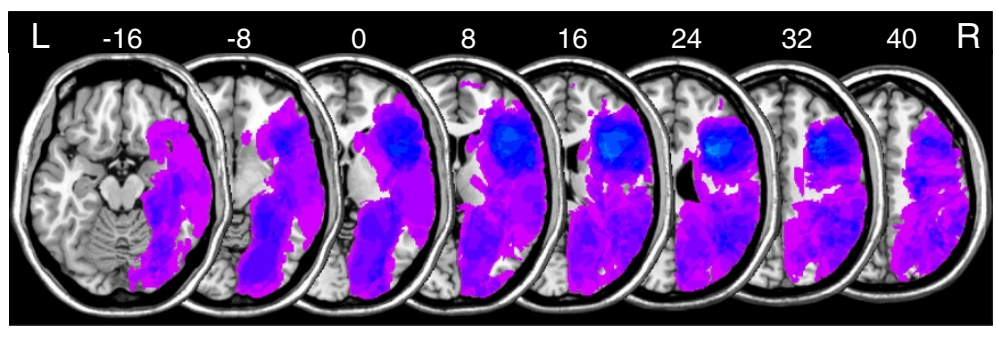

Figure 1 Overlay lesion plots of all thirty-one patients in $M N I$ space. Eight axial slices. The number of overlapping lesions is illustrated by colour, from violet $(N=1)$ to red $(N=31)$. Maximum overlap occurred in the right frontal lobe. The area coloured light blue indicates overlapping lesions in twelve patients (39\% lesion overlap). Numbers indicate MNI coordinates.
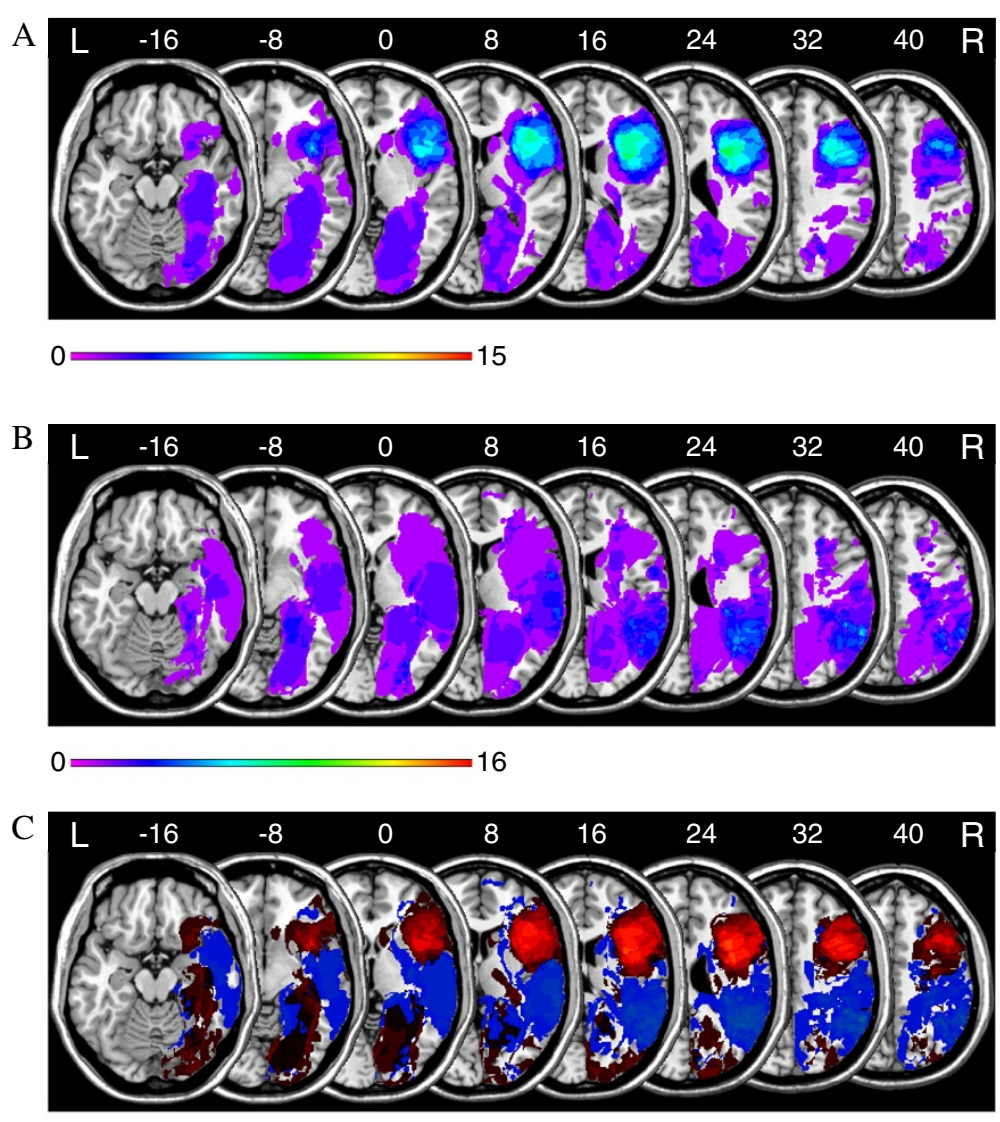

$$
\begin{array}{lllllllllll}
-100 & -80 & -60 & -40 & -20 & 0 & 20 & 40 & 60 & 80 & 100
\end{array}
$$

Figure 2 Anatomical results obtained from the lesion subtraction analysis on the $F A B$ global composite score. A. Overlay lesion plots for those patients who achieved a FAB global composite score below the median ( $M d n=16 ; N=15)$. The number of overlapping lesions is illustrated by colour, from violet $(N=1)$ to red $(N=15)$. B. Overlay lesion plots for those patients who achieved a $F A B$ global composite score equal to or above the median $(M d n=16 ; N=16)$. The number of overlapping lesions is illustrated by colour, from violet $(N=1)$ to red $(N=16)$. C. Overlay plots of the subtracted superimposed lesions of the patients who achieved a $F A B$ global composite score below the median minus patients who achieved a FAB global composite score equal to or above the median. Colours code increasing frequencies from dark red (difference $1 \%$ to $20 \%$ ) to white-yellow (difference $81 \%$ to $100 \%$ ), indicating regions damaged more frequently in patients who achieved a FAB global composite score below the median. The colours from dark blue (difference -1 to $-20 \%$ ) to light green (difference -81 to $-100 \%$ ) indicate regions damaged more frequently in patients who achieved a $F A B$ global composite score equal to or above the median. 
median vs. patients equal to or above the median). The right frontal lobe was more frequently damaged in the group of patients who achieved low $F A B$ global composite scores.

Figure $3 \mathrm{~A}$ depicts the location of those voxels for which the voxel-based lesion-behavior analysis revealed a significant association between voxel damage and the $F A B$ global composite score. This analysis revealed a small area around MNI coordinates $X=35, Y=6, Z=16$, a sub-lobar gray matter coordinate within the anterior insula (BA13).

\section{Lesion analyses: FAB individual task scores}

Figure $3 \mathrm{~B}$ depicts the location of those voxels for which the voxel-based lesion-behavior analysis revealed a significant association between voxel damage and the $F A B$ conceptualization score. Inspection of this map reveals that damage to lateral prefrontal subcortical brain areas is statistically associated with below-median performance in the $F A B$ conceptualization score. Voxel-based statistical analysis revealed three regions: First, an area around MNI coordinates $\mathrm{X}=32, \mathrm{Y}=6, \mathrm{Z}=16$, a sublobar white matter coordinate near the anterior insula (BA13). Second, an area around MNI coordinates $\mathrm{X}=$ $28, Y=15, Z=24$, a sub-gyral white matter coordinate near the claustrum. Third, an area around MNI coordinates $X=37, Y=19, Z=32$, a sub-gyral white matter coordinate underneath the MFG (BA9).

Figure $3 \mathrm{C}$ depicts the location of those voxels for which the voxel-based lesion-behavior analysis revealed a significant association between voxel damage and the $F A B$ mental flexibility score. Inspection of this map reveals that damage to lateral prefrontal subcortical brain areas is statistically associated with below-median performance in the $F A B$ mental flexibility score. Voxelbased statistical analysis revealed an area around MNI coordinates $X=40, Y=20, Z=32$, a white matter coordinate within the right MFG (BA9).

Figure 3D depicts the location of those voxels for which the voxel-based lesion-behavior analysis revealed a significant association between voxel damage and the $F A B$ inhibitory control score. Inspection of this map reveals that damage to lateral prefrontal cortical and subcortical brain areas is statistically associated with belowmedian performance in $F A B$ inhibitory control score. Voxel-based statistical analysis revealed two regions: First, an area around MNI coordinates $X=37, Y=0, Z=16$ (also $X=31, Y=-2, Z=24$ ), sub-lobar white matter coordinates within near the anterior insula (BA13). Second, an area around $\mathrm{MNI}$ coordinates $\mathrm{X}=53-58, \mathrm{Y}=7-18, \mathrm{Z}=8$ 16-24, sub-gyral white matter coordinates underneath the IFG (BA44/45).

The results from the remaining three FAB tasks (programming, sensitivity to interference, environmental autonomy) were negative.

\section{Discussion}

Our voxel-based lesion-behavior mapping data give evidence to the proposition that $F A B$ performance is sensitive to focal frontal lobe damage in the right cerebral hemisphere following stroke. Specifically, several $F A B$ performance indices (i.e., $F A B$ global composite score, $F A B$ conceptualization score, $F A B$ mental flexibility score, and $F A B$ inhibitory control score) are significantly associated with the presence of lateral prefrontal lesions. Even more specifically, we found anatomical correlates of disturbed performance on the $F A B$ global composite score, on the $F A B$ conceptualization score, and on $F A B$ inhibitory control score in or near the anterior insula (BA13). In addition to that, disturbed performance on the $F A B$ mental flexibility score was related to lesions in the MFG (BA9), and performance on the $F A B$ inhibitory control score was sensitive to damage of the right IFG (BA44/45). We did not, however, find evidence for a frontal contribution to performance on the $F A B$ programming, on the $F A B$ sensitivity to interference, and on the $F A B$ environmental autonomy scores. Taken together, our voxel-based lesion-behavior mapping data support the proposition that some, yet not all, $F A B$ measures are sensitive to lateral frontal lobe damage in the right cerebral hemisphere.

No earlier study was published which analyzed the effects of focal brain lesions following stroke on performance indices derived from the $F A B$, despite the fact that demonstrating the sensitivity of any neuropsychological measure to frontal damage is crucial to validating it as a suitable technique for assessing frontal functioning. Our voxel-based lesion-behavior mapping data fill this gap, providing initial evidence for the claim that performance indices on the $F A B$ provide valid measures of frontal dysfunction.

The rapidly-growing literature on the $F A B$ is mainly focused on two issues: First, on its capability to support the early diagnosis and differential diagnosis of neurodegenerative diseases (most notably the early diagnosis of bvFTLD as well as the differential diagnosis of bvFTLD and Alzheimer's disease; [4,23-25]). In this realm, it is worth noting that degenerative brain atrophy affects most notably the anterior insular cortex during the earliest stages of the bvFTLD [26], suggesting that the sensitivity of $F A B$ global performance for early-stage bvFTLD might be attributable, at least in part, to the anatomical association between $F A B$ global composite score, $F A B$ conceptualization score, $F A B$ mental flexibility score, and $F A B$ inhibitory control score and anterior insular dysfunction. Second, the capability of the $F A B$ to detect executive dysfunctions in various diseases affecting fronto-striatal circuits constitutes a recent issue. Specifically, the $F A B$ has been effectually used to document the presence of executive dysfunctions in various neurological diseases (e.g., amyotrophic lateral sclerosis [27,28]; 


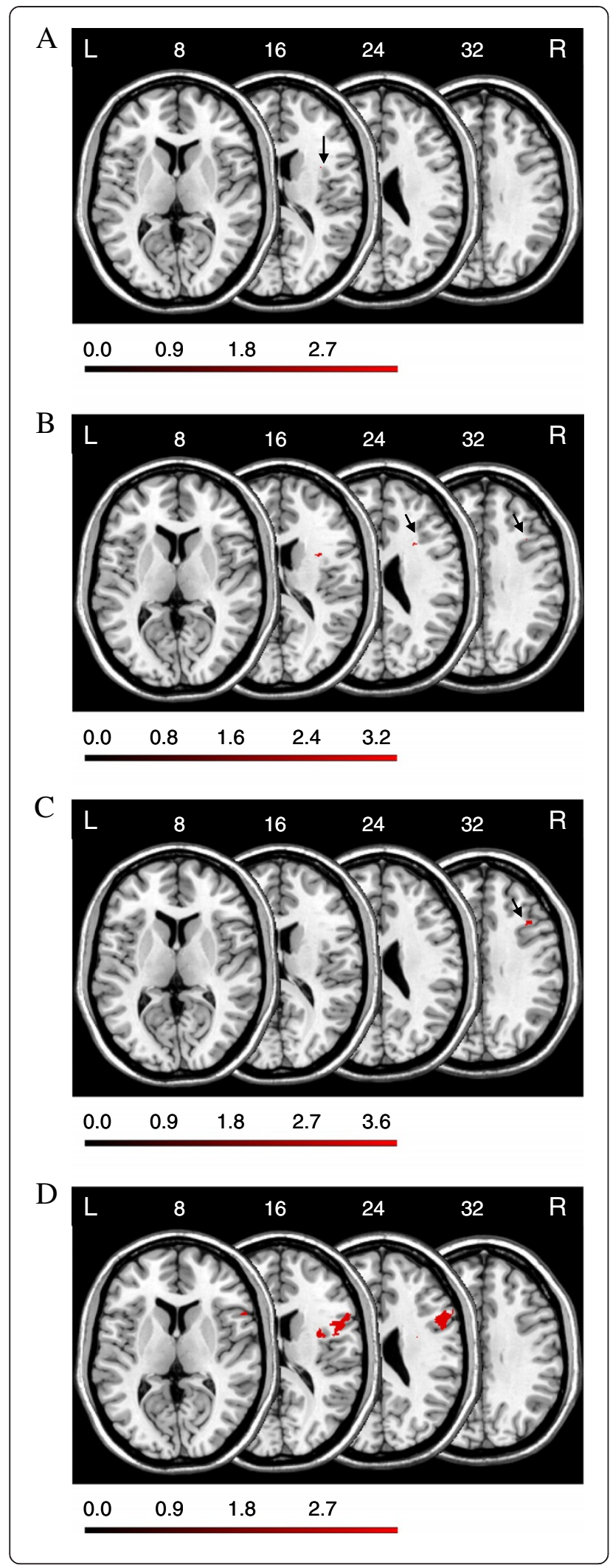

Figure 3 Anatomical results obtained from the voxel-based lesion-behavior mapping (A) on the $F A B$ global composite score, (B) on the $F A B$ conceptualization score, (C) on the $F A B$ mental flexibility score, and (D) on the $F A B$ inhibitory control score. The location of voxels for which the voxel-based lesion-behavior mapping indicated that the observed $L z$ surpassed $z_{\text {crit }}$ is shown. See text for details. Numbers indicate MNI coordinates.

Huntington's disease [29]; multiple system atrophy and progressive supranuclear palsy [30]; Parkinson's disease [31-34]) and psychiatric disorders (e.g., addictive substance abuse [35,36]; depression in Parkinson's disease $[37,38])$. The results of the current study add to this rapidly-growing body of knowledge by strengthening the claim that various indices of $F A B$ performance can be considered as valid assessments of lateral prefrontal, notably anterior insular, functioning.

There are three studies showing relationships between brain perfusion, as assessed by single photon emission computed tomography (SPECT), and FAB performance in patients suffering from various neurodegenerative diseases [39-41]. Although relationships between frontal perfusion and $F A B$ performance have been consistently reported in each of these studies, the exact localization within the frontal lobes as well as the hemispheric lateralization of the anatomical basis of these relationships varied from study to study. A longitudinal study assessed MRI and behavioral measures of disease progression in FTLD [42]. Changes in FAB performance were associated with changes in whole brain MRI atrophy measures, though not uniformly across the three FTLD subgroups (i.e., bvFTLD, semantic dementia, progressive non-fluent aphasia).

Focal injuries to the right anterior insula (BA13) were associated with disturbed performance on the $F A B$ global composite score, on the $F A B$ conceptualization score, and on $F A B$ inhibitory control score. These findings can hardly surprise, given the well-documented capability of the $F A B$ to support the early diagnosis of bvFTLD (see above), and given the already mentioned relationship between degenerative brain atrophy in the anterior insular cortex during the earliest stages of the bvFTLD [24]. Further, anterior insula activations are often observed in functional neuroimaging studies, as detailed below.

The human anterior insular cortex participates in socialemotional processing (e.g., [43]). Other researchers have portrayed it as being part of a hedonic cortical network (e.g., [44]). According to Craig [45], ascending interoceptive pathways terminate in the posterior insula, whereas activation in the anterior insular cortex, possibly organized asymmetrically in an opponent fashion, correlates directly with subjective feelings from the body and with all emotional feelings. Lesions in the right posterior insula are associated with anosognosia for the 
functioning of one's own limbs [46] and with the loss of the sense of limb ownership [47]. The right insular cortex seems to constitute a central node of a network involved in human body scheme representation [48].

The anterior insula/frontal operculum is also known to be involved in some basic cognitive functions. First, the right anterior insula/frontal operculum plays an important role in cognitive control [49-52], and the right anterior insula/frontal operculum seems to be involved in the control over the generation of appropriate behavioral responses to salient stimuli $[53,54]$. Second, activity in the anterior insula is related to the conscious perception of action errors, possibly enabling an orienting response when action errors are detected $[55,56]$. These relationships between activity in the (right) anterior insula and attentional control provide a possible explanation for the observed relationship between lesions in the right anterior insular cortex and $F A B$ inhibitory control scores. Third, performance on tests of fluid intelligence produced extensive activity on the lateral frontal surface, in particular around the inferior frontal sulcus and anterior insula/frontal operculum in functional imaging studies (e.g., [57]), and lesions in these regions are associated with reduced fluid intelligence $[58,59]$. These relationships between activity in the anterior insula/frontal operculum and fluid intelligence provide a possible explanation for the observed relationship between lesions in the right anterior insular cortex and $F A B$ conceptualization scores.

At first glance it may seem surprising that performance on $F A B$ mental flexibility, actually reflecting lexical verbal fluency, was disturbed in stroke patients with injuries in the right frontal lobe. Henry and Crawford [17] reported strong evidence that lexical verbal fluency is more sensitive to frontal than nonfrontal lesions and left as opposed to right cortical lesions. Overall, their results were thus consistent with Ramier and Hecaen's [60] suggestion that lexical verbal fluency performance is mediated by a verbal factor located in the left hemisphere and an executive component that reflects the integrity of the frontal lobes. When viewed from this perspective, the sensitivity of $F A B$ mental flexibility scores to right frontal lesions reflects the degree of integrity of the executive component of lexical verbal fluency. This interpretation is further corroborated by our recent finding that injuries in similar areas of the right frontal lobe (i.e., BA9) are associated with deficient performance accuracy on Form B of the Trail Making Test [61] which requires to continuously switch back and forth between cognitive sets $([62])$.

Further, it has extensively been documented in the literature on imaging and patient studies that the right IFG is closely related to response inhibition (e.g., [63-73]). Our finding contributes to this body of knowledge by showing that performance on the $F A B$ Go - Nogo task, which is a simple clinical assessment technique for the ability to inhibit context-inappropriate responses, is actually sensitive to right IFG lesions in acute stroke patients.

\section{Conclusions}

Our results show that specific aspects of $F A B$ performance can be predicted from the presence of lateral prefrontal lesions, as discussed above. One could express the objection that a biased selection of patients entered the current study. Specifically, most study patients showed prefrontal lesions, whereas only a small number of patients with posterior lesions could be included in our study, thereby biasing the chance to detect reliable brain-behavior relationships in favour of prefrontal regions and to the disadvantage of posterior regions. It is important that we do not wish to claim that the hereby documented sensitivity of performance on the $F A B$ towards prefrontal lesions is specific with regard to this particular lesion location. To date, solid information about the specificity of relationships between performance on the $F A B$ and prefrontal lesions is not available. Another limitation of the current study is the lack of patients with lesions in the left hemisphere, thereby precluding any conclusion on hemispheric asymmetry. As noted by one of the reviewers, poor FAB composite or item scores could localize to areas within the left frontal lobe, but the present data cannot address this possibility.

Our findings are mainly reported in the white matter, while our discussion is essentially addressed on a cortical point of view and in relationship with previous findings in other pathological models. The FAB has formerly been validated on samples of patients with various neurodegenerative syndromes that affect several cortical and subcortical brain structures and white matter tracts. Although there was probably degeneration of frontal cortex in many of these cases, the pathology was clearly not restricted to the frontal cortex, raising the question whether the cognitive impairments observed could be ascribed solely or even primarily to frontal cortex damage. The difficulties in performance on the FAB might have been due to lesions in parts of the brain other than the frontal cortex, including multiple white matter regions. Here, we found disturbed performance on several FAB scores of patients who had damage limited to the frontal cortex and to no more than the immediately subjacent white matter. As it stands now, lesions of white matter subjacent to frontal cortex might be primarily responsible for the observed difficulties in performance on the FAB.

\section{Endnote}

${ }^{\mathrm{a}} \mathrm{A}$ possible statistical solution to the problem would be to use the severity of hemiparesis, apraxia, aphasia, 
pain, hemianopia, neglect and other neuropsychological disturbances as covariates. However, covariance analysis presupposes the separation of patients into meaningful groups of individuals, as in neuropsychological group studies, and it further requires a number of restrictive conditions to be met such as, for example, that the slopes of the regression lines (which relate covariates and dependent variables), fitted to the groups, to be parallel.

\section{Abbreviations}

BA: Brodmann's area; bvFTLD: Behavioural variant of frontotemporal lobar degeneration; CES-D: Center for epidemiologic studies depression scale; CT: Computed tomography; DWI: Diffusion-weighted imaging; EPI: Echo planar imaging; FAB: Frontal assessment battery; FLAIR: Fluid-attenuated inversion-recovery imaging; FOV: Field of view; FTLD: Frontotemporal lobar degeneration; IFG: Inferior frontal gyrus; MCST: Modified card sorting test; MFG: Middle frontal gyrus; MMSE: Mini-mental-state-examination; MNI: Montreal neurological institute; MRI: Magnetic resonance imaging: PFC: Prefrontal cortex; RWT: Regensburger Wortflüssigkeits-test [Regensburger word fluency test]; SPECT: Single photon emission computed tomography; TE: Repetition time; TR: Echo times; VLBM: Voxel-based lesionbehavior mapping; WST: Wortschatz-test [vocabulary test].

\section{Competing interests}

The authors declare that they have no competing interests.

\section{Authors' contributions}

BK contributed to the work by obtaining funding, designing the study, analyzing and interpreting the data, and drafting the manuscript. NR contributed to the work by acquiring and analyzing the data, and drafting the manuscript. ST contributed to the work by acquiring and analyzing the data. HJS contributed to the work by obtaining funding and drafting the manuscript. BdH contributed to the work by analyzing and interpreting the data, and drafting the manuscript. H-OK contributed to the work by obtaining funding, analyzing and interpreting the data, and drafting the manuscript. KW contributed to the work by obtaining funding and drafting the manuscript. All authors read and approved the final manuscript.

\section{Acknowledgements}

BK, NR, ST and KW were supported by the ZNS - Hannelore Kohl Stiftung, Bonn, Germany [grant number 2004007] and by the Erwin-Röver-Stiftung, Hannover, Germany [grant number 20082014]. BdH and H-OK were supported by the Deutsche Forschungsgemeinschaft [grant numbers KA1258/ 15-1, HA 58393/3-1].

\section{Author details}

${ }^{1}$ Cognitive Neurology, Technische Universität Braunschweig, Salzdahlumer Str. 90, Braunschweig 38126, Germany. ${ }^{2}$ Department of Neurology, Braunschweig Hospital, Salzdahlumer Str. 90, Braunschweig 38126, Germany. ${ }^{3}$ Department of Neurology, Hannover Medical School, Carl-Neuberg-Str. 1, Hannover 30625, Germany. ${ }^{4}$ Klinik Niedersachsen, Hauptstr. 59, Bad Nenndorf 31542, Germany. ${ }^{5}$ Division of Neuropsychology, Center of Neurology, Hertie-Institute for Clinical Brain Research, University of Tübingen, Hoppe-Seyler-Str. 3, Tübingen 72076, Germany. ${ }^{6}$ Department of Psychology, University of South Carolina, 915 Greene Street, Columbia SC 29208, USA.

\section{Received: 8 January 2013 Accepted: 30 October 2013}

Published: 16 November 2013

\section{References}

1. Dubois B, Slachevsky A, Litvan I, Pillon B: The FAB: a frontal assessment battery at bedside. Neurology 2000, 55:1621-1626.

2. Luria AR: Higher Cortical Functions in Man. New York: Basic Books; 1966.

3. Lhermitte F, Pillon B, Serdaru M: Human autonomy and the frontal lobes. Part I: imitation and utilization behavior: a neuropsychological study of 75 patients. Ann Neurol 1986, 19:326-334.

4. Slachevsky A, Villapando JM, Sarazin M, Hahn-Barma V, Pillon B, Dubois B: Frontal Assessment battery and differential diagnosis of frontotemporal dementia and Alzheimer disease. Arch Neurol 2004, 61:1104-1107.
5. Seeley WW, Zhou J, Kim EJ: Frontotemporal dementia: what can the behavioral variant teach us about human brain organization? Neuroscientist 2011. Epub Jun 13.

6. Rorden $\mathrm{C}$, Karnath $\mathrm{H}-\mathrm{O}$ : Using human brain lesions to infer function: a relic from a past era in the fMRI age? Nat Rev Neurosci 2004, 5:813-819.

7. Rorden $\mathrm{C}$, Karnath $\mathrm{H}-\mathrm{O}$, Bonilha L: Improving lesion-symptom mapping. J Cogn Neurosci 2007, 19:1081-1088.

8. Rorden C, Fridriksson J, Karnath H-O: An evaluation of traditional and novel tools for lesion behavior mapping. Neuroimage 2009, 44:1355-1362.

9. Stuss DT, Bisschop SM, Alexander MP, Levine B, Katz D, Izukawa D: The trail making test: a study in focal lesion patients. Psychol Assess 2001, 13:230-239

10. Karnath H-O, Steinbach JP: Do brain tumours allow valid conclusions on the localisation of human brain functions? - Objections. Cortex 2011, 47:1004-1006.

11. Radloff LS: The CES-D scale: a self-report depression scale for research in the general population. Appl Psychol Meas 1979, 1:385-401.

12. Oldfield RC: The assessment and analysis of handedness: the Edinburgh inventory. Neuropsychologia 1971, 9:97-113.

13. Folstein MF, Robins $L N$, Helzer JE: The mini-mental state examination. Arch Gen Psychiatry 1983, 40:812

14. Nelson HE: A modified card sorting test sensitive to frontal lobe defects. Cortex 1976, 12:313-324.

15. Aschenbrenner S, Tucher O, Lange KW: Regensburger Wortflüssigkeits-Test [Regensburger Word Fluency Test] (RWT). Göttingen: Hogrefe; 2000.

16. Schmidt K-H, Metzler P: Wortschatztest [Nocabulary Test] (WST). Weinheim: Beltz Test GmbH; 1992

17. Henry JD, Crawford JR: A meta-analytic review of verbal fluency performance following focal cortical lesions. Neuropsychology 2004, 18:284-295.

18. Baldo JV, Schwartz S, Wilkins D, Dronkers NF: Role of frontal versus temporal cortex in verbal fluency as revealed by voxel-based lesion symptom mapping. I Int Neuropsychol Soc 2006, 12:896-900.

19. De Renzi E, Cavalleri F, Facchini S: Imitation and utilisation behaviour. J Neurol Neurosurg Psychiatry 1996, 61:396-400.

20. Brass M, Derrfuss J, Matthes-von Cramon G, von Cramon DY: Imitative response tendencies in patients with frontal brain lesions. Neuropsychology 2003, 17:265-271.

21. Brett $M$, Leff AP, Rorden C, Ashburner J: Spatial normalisation of brain images with focal lesions using cost function masking. Neuroimage 2001, 14:486-500

22. Kimberg DY, Coslett HB, Schwartz MF: Power in voxel-based lesion-symptom mapping. J Cogn Neurosci 2007, 19:1067-1080.

23. Castiglioni S, Pelati O, Zuffi M, Somalvico F, Marino L, Tentorio E, Franceschi M: The frontal assessment battery does not differentiate frontotemporal dementia from Alzheimer's disease. Dement Geriatr Cogn Disord 2006, 22:125-131.

24. Gleichgerrcht E, Roca M, Manes F, Torralva T: Comparing the clinical usefulness of the institute of cognitive neurology (INECO) frontal screening (IFS) and the frontal assessment battery (FAB) in frontotemporal dementia. J Clin Exp Neuropsychol 2011, 33:997-1004.

25. Lipton AM, Ohman KA, Womack KB, Hynan LS, Ninman ET, Lacritz LH: Subscores of the $F A B$ differentiate frontotemporal lobar degeneration from AD. Neurology 2005, 65:726-731.

26. Seeley WW: Anterior insula degeneration in frontotemporal dementia. Brain Struct Funct 2010, 214:465-475.

27. Ahn SW, Kim SH, Kim JE, Kim SM, Sung JJ, Lee KW, Hong YH: Frontal assessment battery to evaluate frontal lobe dysfunction in ALS patients. Can J Neurol Sci 2011, 38:242-246.

28. Oskarsson B, Quan D, Rollins YD, Neville HE, Ringel SP, Arciniegas DE: Using the frontal assessment battery to identify executive function impairments in amyotrophic lateral sclerosis: a preliminary experience. Amyotroph Lateral Scler 2010, 11:244-247.

29. Rodrigues GR, Souza CP, Cetlin RS, de Oliveira DS, Pena-Pereira M, Ujikawa LT, Marques $\mathrm{W} \mathrm{Jr}$, Tumas V: Use of the frontal assessment battery in evaluating executive dysfunction in patients with Huntington's disease. J Neurol 2009, 256:1809-1815.

30. Brown RG, Lacomblez L, Landwehrmeyer BG, Bak T, Utnner I, Dubois B, Agid $Y$, Ludolph A, Bensimon G, Payan C, Leigh NP: Cognitive impairment in patients with multiple system atrophy and progressive supranuclear palsy. Brain 2010, 133:2382-2393. 
31. Koerts J, Tucha L, Leenders KL, van Beilen M, Brouwer WH, Tucha O: Subjective and objective assessment of executive functions in Parkinson's disease. J Neurol Sci 2011, 310:172-175.

32. Lima CF, Meireles LP, Fonseca R, Castro SL, Garrett C: The frontal assessment battery (FAB) in Parkinson's disease and correlations with formal measures of executive functioning. J Neurol 2008, 255:1756-1761.

33. Marconi R, Antonini A, Barone P, Marconi R, Antonini A, Barone P, Colosimo C, Avarello TP, Bottacchi E, Cannas A, Ceravolo MG, Ceravolo R, Cicarelli G, Gaglio RM, Giglia L, lemolo F, Manfredi M, Meco G, Nicoletti A, Pederzoli M, Petrone A, Pisani A, Pontieri FE, Quatrale R, Ramat S, Scala R, Volpe G, Zappulla S, Bentivoglio AR, Stocchi F, et al: Frontal assessment battery scores and non-motor symptoms in parkinsonian disorders. Neurol $\mathrm{SCi}$ 2012, 33:585-593.

34. Santangelo R, Vitale C, Trojano L, Verde F, Grossi D, Barone P: Cognitive dysfunctions and pathological gambling in patients with Parkinson's disease. Mov Disord 2009, 24:899-905.

35. Cunha PJ, Nicastri S, de Andrade AG, Bolla KL: The frontal assessment battery (FAB) reveals neurocognitive dysfunction in substance-dependent individuals in distinct executive domains: abstract reasoning, motor programming, and cognitive flexibility. Addict Behav 2010, 35:875-881.

36. Fontes MA, Bolla Kl, Cunha PJ, Almeida PP, Jungerman F, Laranjeira RR, Bressan RA, Lacerda AL: Fontal assessment battery (FAB) is a simple tool for detecting executive deficits in chronic cannabis users. $J$ Clin Exp Neuropsychol 2011, 10:1-9.

37. Kummer A, Harsányi E, Dias FM, Cardoso F, Caramelli P, Teixeira AL: Depression impairs executive functioning in Parkinson disease patients with low educational level. Cogn Behav Neurol 2009, 22:167-172.

38. Santangelo R, Vitale C, Trojano L, Longo K, Cozzolino A, Grossi D, Barone P: Relationship between depression and cognitive dysfunctions in Parkinson's disease without dementia. J Neurol 2009, 256:632-638.

39. Guedj E, Allali G, Goetz C, Le Ber I, Volteau M, Lacomblez L, Vera P, Hitzel A Hannequin D, Decousus M, Thomas-Antérion C, Magne C, Vercelletto M, Bernard AM, Didic M, Lotterie JA, Puel M, Brice A, the French research network on FTD/FTD-MND, Habert M-O, Dubois B: Frontal assessment battery is a marker of dorsolateral and medial frontal functions: a SPECT study in frontotemporal dementia. J Neurol Sci 2008, 273:84-87.

40. Yoshida H, Terada S, Sato S, Kishimoto Y, Ata T, Ohshima E, Honda H, Ishihara T, Kuroda S: Frontal assessment battery and brain perfusion imaging in early dementia. Dement Geriatr Cogn Disord 2009, 27:133-138.

41. Kume K, Hnyu H, Murakami M, Sato T, Hirao K, Kanetaka H, Sakurai H, Iwamoto T: Frontal assessment battery and brain perfusion images in amnestic mild cognitive impairment. Geriatr Gerontol Int 2011, 11:77-82.

42. Gordon E, Rohrer JD, Kim LG, Omar R, Rossor MN, Fox NC, Warren JD: Measuring disease progression in frontotemporal lobar degeneration: a clinical and MRI study. Neurology 2010, 74:666-673.

43. Singer $T$, Critchley HD, Preuschoff $K$ : A common role of insula in feelings, empathy and uncertainty. Trends Cogn Sci 2009, 13:334-340.

44. Kringelbach ML, Berridge KC: Towards a functional neuroanatomy of pleasure and happiness. Trends Cogn Sci 2009, 13:479-487.

45. Craig AD: Significance of the insula for the evolution of human awareness of feelings from the body. Ann N Y Acad Sci 2011, 1225:72-82.

46. Karnath $\mathrm{H}-\mathrm{O}$, Baier B, Nägele T: Awareness of the functioning of one's own limbs mediated by the insular cortex? J Neurosci 2005, 25:7134-7138.

47. Baier B, Karnath H-O: Tight link between our sense of limb ownership and self-awareness of actions. Stroke 2008, 39:486-488.

48. Karnath $\mathrm{H}-\mathrm{O}$, Baier B: Right insula for our sense of limb ownership and self-awareness of actions. Brain Struct Funct 2010, 214:411-417.

49. Derrfuss J, Brass M, von Cramon DY: Cognitive control in the posterior frontolateral cortex: evidence from common activations in task coordination, interference control, and working memory. Neuroimage 2004, 23:604-612.

50. Derrfuss J, Brass M, Neumann J, von Cramon DY: Involvement of the inferior frontal junction in cognitive control: meta-analyses of switching and stroop studies. Hum Brain Mapp 2005, 25:22-34

51. Dosenbach NU, Visscher KM, Palmer ED, Miezin FM, Wenger KK, Kang HC, Burgund ED, Grimes AL, Schlaggar BL, Petersen SE: A core system for the implementation of task sets. Neuron 2006, 50:799-812.

52. Nelson SM, Dosenbach NUF, Cohen AL, Wheeler ME, Schlaggar BL, Petersen SE: Role of the anterior insula in task-level control and focal attention. Brain Struct Funct 2010, 214:669-680.
53. Eckert MA, Menon V, Walczak A, Ahlstrom J, Denslow S, Horwitz A, Dubno JR: At the heart of the ventral attention system: the right anterior insula. Hum Brain Mapp 2009, 30:2530-2541.

54. Menon V, Uddin LQ: Saliency, switching, attention and control: a network model of insula function. Brain Struct Funct 2010, 214:655-667.

55. Ramautar JR, Slagter HA, Kok A, Ridderinkhof KR: Probability effects in the stop-signal paradigm: the insula and the significance of failed inhibition. Brain Res 2006, 11:143-154.

56. Ullsperger M, Harsay HA, Wessel JR, Ridderinkhof KR: Conscious perception of errors and its relation to the anterior insula. Brain Struct Funct 2010, 214:629-643.

57. Duncan J, Seitz RJ, Kolodny J, Bor D, Herzog H, Ahmed A, Newell FN, Emslie H: A neural basis for general intelligence. Science 2000, 289:457-460.

58. Roca M, Parr A, Thompson R, Woolgar A, Torralva T, Antoun N, Manes F, Duncan J: Executive function and fluid intelligence after frontal lobe lesions. Brain 2010, 133:234-247.

59. Woolgar A, Parr A, Cusack R, Thompson R, Nimmo-Smith I, Torralva T, Roca M, Antoun N, Manes F, Duncan J: Fluid intelligence loss linked to restricted regions of damage within frontal and parietal cortex. Proc Natl Acad SCi USA 2010, 107:14899-14902.

60. Ramier AM, Hécaen H: Role respectif des atteintes frontales et de la lateralisation lesionnellle dans les deficits de la "fluence verbale" [Respective rôles of frontal lesions and lesion lateralization in "verbal fluency" deficiencies]. Rev Neurol (Paris) 1970, 123:17-22.

61. Reitan RM: Trail Making Test: Manual for administration and scoring. Tucson, AZ: Reitan Neuropsychology Laboratory; 1992.

62. Kopp B, Rösser N, Wessel K: Psychometric characteristics and practice effects of the Brunswick trail making test. Percept Mot Skills 2008, 107:707-733.

63. Aron AR: The neural basis of inhibition in cognitive control. Neuroscientist 2007, 13:214-228.

64. Aron AR: From reactive to proactive and selective control: developing a richer model for stopping inappropriate responses. Biol Psychiatry 2011, 69:55-68.

65. Aron AR, Monsell S, Sahakian BJ, Robbins TW: A componential analysis of task-switching deficits associated with lesions of left and right frontal cortex. Brain 2004, 127:1561-1573.

66. Aron AR, Robbins TW, Poldrack RA: Inhibition and the right inferior frontal cortex. Trends Cogn Sci 2004, 8:170-177.

67. Brass M, Derrfuss J, Forstmann B, von Cramon DY: The role of the inferior frontal junction area in cognitive control. Trends Cogn Sci 2005, 9:314-316.

68. Brass $M$, Haggard P: To do or not to do: the neural signature of self-control. J Neurosci 2007, 27:9141-9145

69. Brass M, Haggard P: The hidden side of intentional action: the role of the anterior insular cortex. Neuropsychologia 2010, 43:89-98.

70. Chikazoe J, Jimura K, Asari T, Yamashita K, Morimoto H, Hirose S, Miyashita Y, Konishi S: Functional dissociation in right inferior frontal cortex during performance of Go/No-Go task. Cereb Cortex 2009, 19:146-152.

71. Picton TW, Stuss DT, Alexander MP, Shallice T, Binns MA, Gillingham S: Effects of focal frontal lesions on response inhibition. Cereb Cortex 2007, 17:826-838.

72. Robbins TW: Shifting and stopping: fronto-striatal substrates, neurochemical modulation and clinical implications. Philos Trans R Soc Lond B Biol Sci 2007, 362:917-932

73. Xue G, Aron AR, Poldrack RA: Common neural substrates for inhibition of spoken and manual responses. Cereb Cortex 2008, 18:1923-1932.

doi:10.1186/1471-2377-13-179

Cite this article as: Kopp et al:: Performance on the Frontal Assessment Battery is sensitive to frontal lobe damage in stroke patients. BMC Neurology 2013 13:179. 